RICYDE. Revista Internacional de Ciencias del Deporte doi: $10.5232 /$ ricyde

Rev. int. cienc. deporte

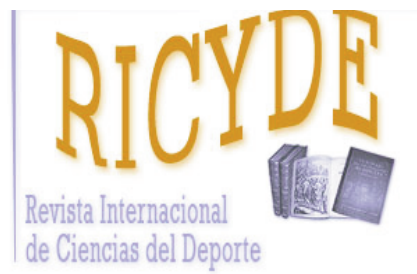

RICYDE. Revista Internacional de Ciencias del Deporte VOLUMEN XIV - AÑO XIV

Paginas:163-173 ISSN:1885-3137

Número: 52 - Abril - 2018

\title{
Contribución de los brazos en el aterrizaje del salto vertical Contribution of the arms in the landing of the vertical jump
}

\author{
Marcos Gutiérrez-Dávila, David Pancorbo, Jesús Olivares, Francisco Javier Rojas \\ Departamento de Educación Física y Deportiva. Universidad de Granada. España
}

\section{Resumen}

Se pretende comprobar el efecto que tiene la contribución de los brazos en los aterrizajes de los saltos verticales sobre las componentes de las fuerzas de reacción, goniometría articular y contribución de los segmentos corporales al desplazamiento vertical del centro de gravedad (CG). Han participado 29 deportistas donde el salto vertical constituye una habilidad básica. Todos los participantes debían dejarse caer desde una altura de $0.5 \mathrm{~m}$ y amortiguar la caída en dos situaciones experimentales: a) sin acción de brazos y b) con participación libre de los brazos. Se ha utilizado una plataforma de fuerza, operando a 500 $\mathrm{Hz}$, sincronizada temporalmente a una cámara de vídeo a $240 \mathrm{~Hz}$ que registraba el plano sagital de los saltos. Los saltos han sido considerados como un movimiento simétrico que se desarrolla en un plano, compuesto por un modelo mecánico simplificado de ocho segmentos. Los resultados han puesto de manifiesto que la participación libre de los brazos contribuye a reducir del segundo pico máximo de fuerza, lo que se produce debido a la mayor distancia de frenado del CG y una menor reducción de la velocidad vertical del CG, lo que podría contribuir a reducir el riesgo de lesiones. La contribución segmentaria al desplazamiento vertical del CG indica que la restricción de los brazos durante los aterrizajes produce un cambio importante en el mecanismo de absorción de las fuerzas que podría incrementar la tensión en el ligamento cruzado anterior de la rodilla.

Palabras clave: Biomecánica; recepción salto vertical; amortiguación; lesiones deportivas.

\section{Resumen}

The purpose was to evaluate the effect of the arms action during vertical jump landing on ground reaction forces, joint kinematic and vertical displacement of the center of gravity (CG). 29 athletes where the vertical jump constitutes a basic skill in their sport have participated in this study. All participants had to drop from a height of $0.5 \mathrm{~m}$ and cushion the fall in two experimental situations: a) without arm action and b) with free participation of the arms. A force platform, operating at $500 \mathrm{~Hz}$, was synchronized temporarily to a video camera at $210 \mathrm{~Hz}$ which recorded the sagittal plane of the jumps. The jumps have been considered as a symmetrical movement that develops in a plane, composed by a simplified mechanical model of eight segments. The results have shown that the free participation of the arms contributes to reduce the second peak of force, which is due to the greater braking distance of the CG and a smaller reduction of the vertical velocity of the CG, which could contribute to reducing the risk of injury. The segmental contribution to the vertical displacement of the CG indicates that the restriction of the arms during landings produces a significant change in the mechanism of absorption of the forces that could increase the tension in the anterior cruciate ligament of the knee.

Key words: Biomechanics; vertical jump landing; cushioning, sports injuries. 
Gutiérrez-Dávila, M.; Pancorbo, D.; Olivares, J., y Rojas, F. J. (2018). Contribución de los brazos en el aterrizaje del salto vertical. RICYDE. Revista internacional de ciencias del deporte, 52(14), 163-173. https://doi.org/10.5232/ricyde2018.05206

\section{Introducción}

$\mathrm{P}$ osiblemente la batida del salto vertical sea uno de los gestos técnicos más estudiados en todas sus manifestaciones o estilos, orientándose la mayoría de esas investigaciones hacia la búsqueda de soluciones para mejorar el impulso vertical y no tanto al estudio de la absorción de las fuerzas durante el aterrizaje posterior. Sin embargo, debido a su relación con ciertas lesiones deportivas, en los últimos años ha surgido un interés creciente por su estudio (Cortes, Onate, Abrantes, Gagen, Dowling, y Van Lunen, B, 2007; Decker, Torry, Wyland, Sterett y Steadman, 2003; Ericksen, Gribble, Pfile y Pietrosimone, 2013).

Efectivamente, investigaciones previas han puesto de manifiesto que ciertas lesiones deportivas producidas sin contacto, están asociadas a la escasa amortiguación durante la recepción de los saltos (Cortes y col., 2007; Chappell, Yu, Kirkendall y Garret, 2002; Rowley y Richards, 2015; Wilk, Briem, Reinold, Devine, Dugas y Andrews, 2006), constatándose que una mejora en la técnica de aterrizaje podría reducir el riesgo de lesiones (Eriksen y col., 2013, Lobietti, Coleman, Pizzichillo y Merni, 2010). Concretamente, ciertas fracturas por estrés se han asociado con las repetidas tensiones que producen los impactos en el sistema músculo esquelético (Newman y Newberg, 2010; Rojano, Rodríguez y Berral, 2010; Rowley y Richards, 2015) o, lesiones tan importantes como las producidas en el ligamento cruzado anterior, parecen estar favorecidas por ciertos factores extrínsecos como el incremento de las cargas de impacto, pequeños ángulos en valgo de la rodilla, el desplazamiento de la tibia hacia delante o la falta de coordinación en la recepción (Chappell y col., 2002; Ericksen y col., 2013; Gutiérrez-Dávila, Olivares, Pancorbo y Rojas, 2017; McNair y Marshall, 1994).

Del análisis de las fuerzas de reacción que se producen durante la mayoría de los aterrizajes, se hace referencia a una fase de absorción del impacto que estaría comprendida entre los primeros 150 y $200 \mathrm{~ms}$ a partir del primer contacto con el suelo (Lees, 1981). En los primeros $100 \mathrm{~ms}$ de esta fase, se suelen observar dos picos de fuerza: a) uno producido por el impacto de la parte anterior del pie (1-PMF) y b) Un segundo pico producido por el aterrizaje de la parte posterior del pie (2-PMF), siendo éste el que parece estar más asociado la producción de lesiones (Cámara, Calleja-González, Martínez y Fernández-López, 2013; Decker et al., 2003; McNair, Prapavessis y Callender, 2000; Rojano, Rodríguez, Berral, 2010). Aunque no se ha podido constatar que la reducción del 2-PMF contribuya a reducir el riesgo de lesiones, los datos sugieren esta hipótesis, especialmente cuando se asocia a momentos articulares debidos a ciertas posiciones segmentarias que alejan a las articulaciones de la dirección de la fuerza de reacción resultante.

Los aterrizajes están controlados por un complejo sistema neural y reflejo que permite modular las fuerzas musculares antes del contacto y durante toda la fase de absorción del impacto (Sampello, 2016). De este modo, aunque se conoce que las articulaciones de la rodilla y el tobillo son las máximas responsables de la amortiguación de las fuerzas durante los aterrizajes (Decker et al., 2003), es necesario considerar que este sistema motor posee un control global que desemboca en una acción coordinativa de todo el cuerpo (McNintt-Gray, 2000), donde los brazos actúan de forma sincronizada contribuyendo a la amortiguación de las fuerzas. En este sentido, Dapena y Chung (1988) describieron la acción de los brazos hacia abajo y hacia delante, durante el impacto inicial de la batida del salto de altura, como un amortiguador de las fuerza de reacción y Niu, Zhang y Zhao (2013) han sugerido que una adecuada coordinación de los brazos hace que se reduzca el pico de fuerza durante el impacto inicial, que aumente la distancia de frenado y contribuyan a la estabilidad del aterrizaje.

Además de la aportaciones señaladas, teorías debidamente justificadas permiten sugerir tres aspectos positivos de la acción de brazos en la absorción de los impactos durante la recepción 
Gutiérrez-Dávila, M.; Pancorbo, D.; Olivares, J., y Rojas, F. J. (2018). Contribución de los brazos en el aterrizaje del salto vertical. RICYDE. Revista internacional de ciencias del deporte, 52(14), 163-173. https://doi.org/10.5232/ricyde2018.05206

de los saltos verticales: a) En los aterrizajes con doble apoyo se ha constatado que, al aumentar la altura de caída, se incrementa el pico máximo de la fuerza de reacción (Niu, Feng, Jiang y Zhang, 2014), posiblemente debido a una mayor velocidad vertical del centro de gravedad (CG) en el instante de tomar contacto con el suelo. Sin embargo, en sistemas coordinados de $n$ segmentos, como es el cuerpo humano, la velocidad vertical está determinada por el sumatorio del producto de las masas segmentarias y sus respectivas velocidades verticales dividido por la masa total del sistema. Así, en el instante de tomar contacto con el suelo, una acción de los brazos hacia abajo, mediante una extensión de hombros, haría disminuir menos la velocidad vertical del resto del cuerpo y, en consecuencia, también el pico máximo de fuerza. b) Partiendo de este mismo sistema coordinado del cuerpo humano, la desaceleración producida por el CG durante los aterrizajes está relacionada con el sumatorio del producto de las masas segmentarias y sus respectivas aceleraciones dividido por la masa total. Así, la aceleración de los brazos hacia abajo, mediante una extensión de hombros, durante la fase de absorción del impacto reduciría la desaceleración del resto del cuerpo $\mathrm{y}$, en consecuencia, también la fuerza de reacción que producen dichas desaceleraciones y c) Partir de una posición alta de los brazos en el instante de tomar contacto con el suelo y mantener en éstos cierta velocidad vertical hasta el final del aterrizaje, incrementará la distancia de frenado del CG, lo que contribuiría a reducir los picos de fuerza.

Según lo expuesto, en esta investigación se pretende comprobar el efecto que tiene la contribución de los brazos sobre los factores que determinan la amortiguación de los saltos verticales, exponiéndose como hipótesis que la acción de los brazos reducen el pico máximo de fuerza y aumentan la distancia de frenado.

\section{Método}

\section{Participantes}

Han participado 29 estudiantes hombres de la Facultad de Ciencias del Deporte (edad $=21.1$ \pm 1.7 años; talla $=1.78 \pm 0.06 \mathrm{~m}$; masa $=70.6 \pm 8.1 \mathrm{~kg}$ ), utilizando para su selección el criterio de haber participado de forma regular en actividades deportivas relacionadas con el salto vertical. A todos ellos se les informó y solicitó su consentimiento para participar en este estudio siguiendo las directrices de la Comisión Ética de la Universidad.

\section{Material y procedimientos}

Se ha utilizado una plataforma de fuerza Dinascan/IBV, operando a $500 \mathrm{~Hz}$, sincronizada temporalmente a una cámara de vídeo Casio EX - FH20, que registraba a $240 \mathrm{~Hz}$ el plano sagital del movimiento. Después de un calentamiento normalizado de diez minutos consistente en carrera, ejercicios de movilidad articular y saltos, los participantes realizaron los ensayos necesarios hasta familiarizarse con los sistemas de registro y las dos condiciones de ejecución propuestas. Así, en la situación Sin brazos, debían dejarse caer a la Plataforma desde una altura de $0.5 \mathrm{~m}$, partiendo de una posición erguida con las manos en las caderas, manteniéndolas en esa posición durante toda la recepción. En la situación "Con brazos" se realizaba este mismo movimiento aunque partiendo con los brazos por encima de la cabeza para bloquear la altura del CG y el rango de movimiento vertical de los brazos y permitiendo su participación libre durante el vuelo y el aterrizaje (Figura 1). A continuación, los participantes realizaron dos series de 5 saltos válidos (una para cada situación experimental) con una recuperación de 2 minutos entre ensayo y de 10 minutos entre serie. El orden de las series fue alterado entre los participantes. Posteriormente sólo se analizó el ensayo cuyo tiempo para frenar el movimiento descendente del CG era el mediano entre los cinco de cada situación experimental. 
Gutiérrez-Dávila, M.; Pancorbo, D.; Olivares, J., y Rojas, F. J. (2018). Contribución de los brazos en el aterrizaje del salto vertical. RICYDE. Revista internacional de ciencias del deporte, 52(14), 163-173. https://doi.org/10.5232/ricyde2018.05206

\section{Análisis de datos}

Para cada ensayo se determinó el posible error sistemático procedente de la plataforma de fuerzas a parir de la media de las componentes horizontal y vertical $\left(\mathbf{F}_{\mathrm{R}(\mathrm{X})}\right.$ y $\mathbf{F}_{\mathrm{R}(\mathrm{Y})}$, respectivamente) de los 20 registros anteriores a la toma de contacto (Figura 1). Después de restar el posible error sistemático y el peso del sujeto para la componente vertical, se registró el valor máximo del segundo pico de fuerza vertical que surge en los instantes iniciales de la fase de absorción del impacto (2-PMF). La aceleración horizontal y vertical del CG se determinaron a partir de $\mathbf{F}_{\mathrm{R}(\mathrm{X})}$ y $\mathbf{F}_{\mathrm{R}(\mathrm{Y})}$ y la masa del saltador. Finalmente, los sucesivos registros de las componentes de la velocidad del CG $\left(\mathbf{v}_{(\mathrm{X}) \mathrm{CG}} \mathrm{y} \mathbf{v}_{(\mathrm{Y}) \mathrm{CG}}\right.$, respectivamente) y de las posiciones $\left(\mathbf{S}_{(\mathrm{X}) \mathrm{CG}}\right.$ y $\mathbf{S}_{(\mathrm{Y}) \mathrm{CG}}$, respectivamente), se determinaron mediante integración de sus respectivas funciones de aceleración-tiempo y velocidad-tiempo, respectivamente, usando para ello el método trapezoidal con un incremento temporal de $0.002 \mathrm{~s}$. Las constantes de integración se han obtenido a partir de la digitalización manual de las imágenes de vídeo.

Para simplificar el procedimiento de digitalización, los aterrizajes se han considerado como un movimiento simétrico que se desarrolla en el plano, lo que nos ha permitido utilizar un modelo coordinado simplificado de 8 segmentos, definidos por 12 marcadores situados en el cuerpo del saltador (Extremos anterior y posterior del pie; centros articulares del tobillo, rodilla, cadera, hombro, codo y muñeca; $3^{\circ}$ metacarpiano de la mano; hueco supraexternal; ángulo del maxilar y vertex). La posición de los marcadores se estableció según el modelo y los parámetros inerciales propuestos por Zatsiorsky y Seluyanov (1985) y adaptados por de Leva (1996). En este modelo simplificado, la masa de los segmentos correspondientes a las extremidades, se han considerado como la suma de los dos segmentos correspondientes. Tras la digitalización, las coordenadas planas fueron suavizadas mediante un filtro digital de paso bajo a $8 \mathrm{~Hz}$ (Winter, 1990) e interpoladas a $500 \mathrm{~Hz}$, utilizando splines de quinto grado. La conversión de las coordenadas digitalizadas en datos reales se realizó a partir de un sistema de referencia que consistía en un cubo de $2 \times 2 \times 0.5 \mathrm{~m}$ centrado con respecto al lugar de la recepción. Las constantes de integración correspondientes la posición del CG, en el momento aproximado del contacto con la plataforma (entre dos imágenes), se determinó con respecto el centro geométrico de los dos apoyos cuando estos estaban plenamente apoyados en la plataforma y las componentes de la velocidad instantánea del CG se determinaron mediante la primera derivada de la posición vertical del CG con respecto al tiempo.

A partir de las coordenadas planas del modelo, se determinaron las sucesivas posiciones angulares de las articulaciones de la cadera, rodilla y tobillo, utilizando para ello el producto escalar de los vectores que definían las posiciones de sus respectivos segmentos. Es necesario aclarar que el segmento pie se definió como el vector que une los puntos del centro articular del tobillo y el final del pie. Siguiendo la metodología propuesta por Gutiérrez-Dávila, Garrido, Amaro, Ramos y Rojas (2012), esas mismas coordenadas fueron utilizadas para determinar la contribución de los segmentos al desplazamiento vertical del CG durante la fase de absorción del impacto, definida por los primeros $200 \mathrm{~ms}$ desde el contacto con la plataforma (Lees, 1981).

Para evaluar la fiabilidad de las pruebas, se ha aplicado un análisis de varianza de medidas repetidas a todos los ensayos en las dos condiciones experimentales (cinco ensayos para cada condición), tomando como variable dependiente el tiempo de absorción total, no existiendo diferencias significativas entre los ensayos. El coeficiente de correlación intraclase para esta misma variable, ha sido de $0.84(\mathrm{p}<0.001)$ para la condición sin acción de brazos y 0.78 $(\mathrm{p}<0.001)$ para la condición con participación libre de los brazos. Se ha calculado la media y desviación típica para cada variable en cada situación experimental, determinándose las 
Gutiérrez-Dávila, M.; Pancorbo, D.; Olivares, J., y Rojas, F. J. (2018). Contribución de los brazos en el aterrizaje del salto vertical. RICYDE. Revista internacional de ciencias del deporte, 52(14), 163-173. https://doi.org/10.5232/ricyde2018.05206

diferencias entre las medias de los dos niveles de la variable mediante un análisis de varianza de medidas repetidas (ANOVA). El nivel de significación se ha establecido en $\mathrm{p}<0.05$. El tamaño el efecto de las diferencias entre el salto con y sin brazos fue calculado mediante el coeficiente " $\mathrm{d}$ " de Cohen (1988). La magnitud de las diferencias fue considerada trivial $(\mathrm{d}<$ $0.2)$, pequeña $(0.2 \leq \mathrm{d}<0.5)$, moderada $(0.5 \leq \mathrm{d}<0.8)$ y grande $(\mathrm{d} \geq 0.8)$ Se ha utilizado el paquete estadístico Statgraphic Plus 5.1 para Windows.

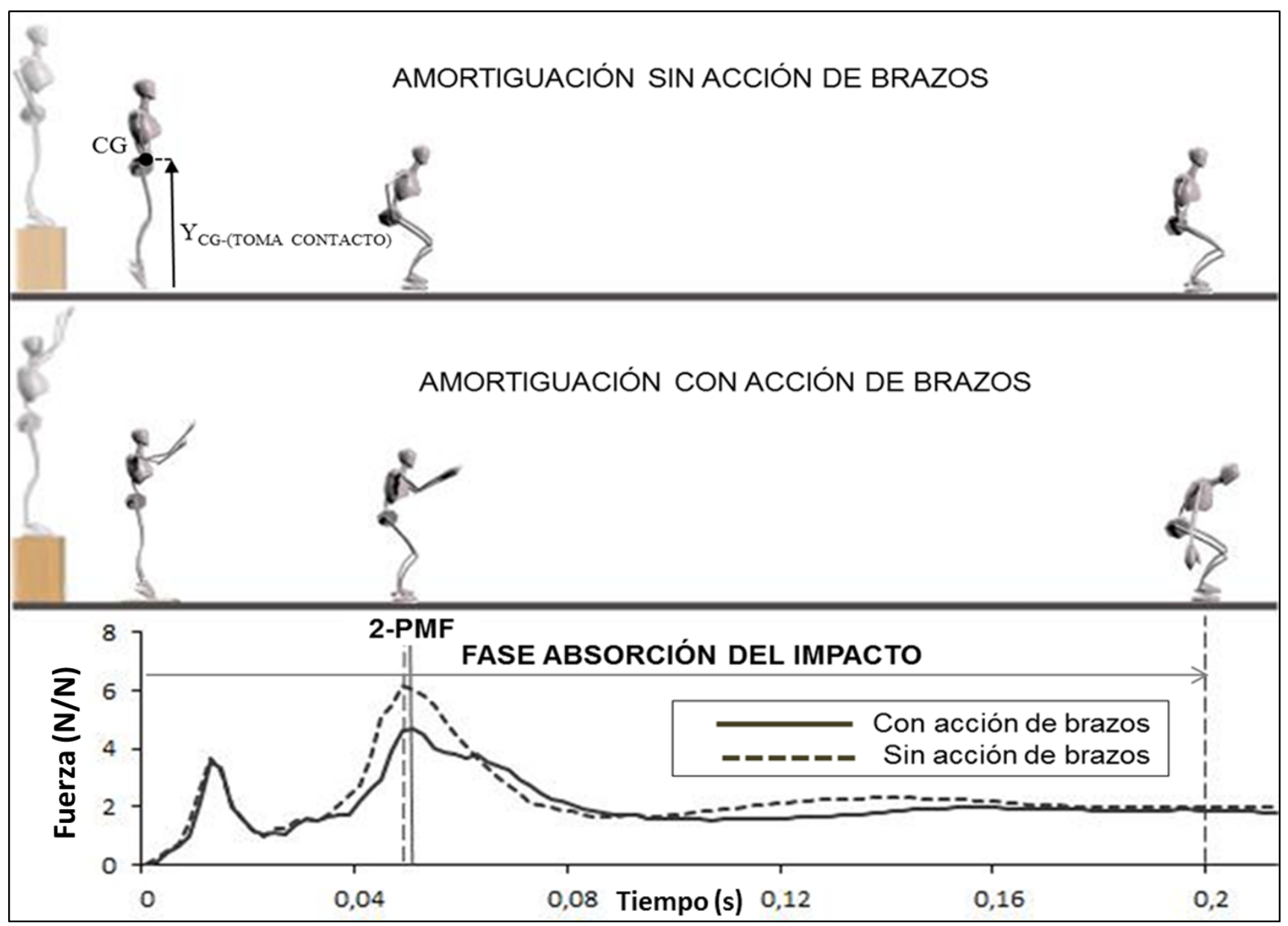

Figura 1.- Representación de la amortiguación sin acción de brazos, con acción de brazos y gráfica fuerza vertical-tiempo de las dos situaciones

\section{Resultados}

En la tabla 1 se presenta una estadística descriptiva e inferencial de las variables generales relacionadas con la amortiguación para las dos situaciones experimentales: a) amortiguación de la caída sin acción de brazos y b) amortiguación con acción de brazos. Los datos constatan que, con la participación de los brazo, el segundo pico máximo de la fuerza vertical (2-PMF) se reduce significativamente $(\mathrm{p}<0.01)$, sin que hayan existido diferencias estadísticamente significativas en el tiempo medio requerido para alcanzar 2-PMF (t-2-PMF). Los datos también confirman la hipótesis inicial al constatarse que la altura del CG en la toma de contacto se incrementa de forma muy significativa cuando se utilizan los brazos $(\mathrm{p}<0.001)$. La estadística inferencial aplicada a la goniometría del tobillo, rodilla y cadera en el instante de tomar contacto con la plataforma, sólo nos ha permitido constatar diferencias entre las medias para la articulación de la cadera $(\mathrm{p}<0.01)$, siendo significativamente mayor su ángulo cuando los aterrizajes se realizan con acción de brazos. 
Gutiérrez-Dávila, M.; Pancorbo, D.; Olivares, J., y Rojas, F. J. (2018). Contribución de los brazos en el aterrizaje del salto vertical. RICYDE. Revista internacional de ciencias del deporte, 52(14), 163-173. https://doi.org/10.5232/ricyde2018.05206

Tabla 1.- Estadística descriptiva e inferencial de las variables generales relacionadas con la amortiguación de la caída para las dos condiciones experimentales. Los valores de la fuerza se han expresado en Newton de fuerza por Newton de peso corporal.

\begin{tabular}{|c|c|c|c|c|}
\hline variables & sin brazos & con brazos & f & Cohen's d \\
\hline 2-PMF (N/N) & $5.42 \pm 1.32$ & $4.94 \pm 1.34$ & $7.88^{* *}$ & 0.4 \\
\hline Instante del 2-PMF (s) & $0.046 \pm 0.010$ & $0.050 \pm 0.015$ & 3.42 & 0.4 \\
\hline $\mathbf{Y}_{\text {CG-(TOMA CONTACTO) }}(\mathrm{m})$ & $1.106 \pm 0.040$ & $1.154 \pm 0.041$ & $107.98^{* * *}$ & 1.2 \\
\hline $\mathbf{X}_{\text {CG-(TOMA CONTACTO) }}(\mathrm{m})$ & $-0.068 \pm 0.027$ & $-0.068 \pm 0,018$ & 0.01 & 0 \\
\hline $\boldsymbol{\theta}$ TOBILLO-(TOMA CONTACTO) $\left({ }^{\circ}\right)$ & $134 \pm 8$ & $134 \pm 7$ & 0.22 & 0 \\
\hline $\boldsymbol{\theta}_{\text {RODILLA-(TOMA CONTACTO) }}\left({ }^{\circ}\right)$ & $159 \pm 7$ & $159 \pm 6$ & 0.90 & 0 \\
\hline $\boldsymbol{\theta}$ CADERA-(TOMA CONTACTO) $\left({ }^{\circ}\right)$ & $152 \pm 6$ & $157 \pm 6$ & $10.74^{* *}$ & 0.8 \\
\hline
\end{tabular}

2-PMF (N/N) Segundo pico máximo de fuerza, se expresa en Newton divididos entre el peso de cada participante

${ }^{* * *} p<0.001 ;{ }^{* *} p<0.01 ;{ }^{*} p<0.05$

En la tabla 2 se presenta una estadística descriptiva e inferencial de las componentes del desplazamiento del CG hasta que se alcanza el segundo pico máximo de fuerza y durante la fase de absorción del impacto que, como se había descrito, se producen durante los $200 \mathrm{~ms}$ tras la toma de contacto con la plataforma. Los datos indican que existe una cierta significación entre las medias del desplazamiento vertical del CG hasta que se alcanza el segundo pico máximo de fuerza $(\mathrm{p}<0.05)$, siendo el desplazamiento vertical del CG mayor cuando los aterrizajes se realizan con acción de brazos. Al final de fase de absorción del impacto se incrementan las diferencias entre la medias en el desplazamiento vertical del CG $(\mathrm{p}<0.01)$. Con respecto al desplazamiento horizontal medio del CG, sólo han existido ciertas diferencias al final de esta fase $(\mathrm{p}<0.05)$, sido menor con la participación de brazos. En esta misma tabla se presentan los datos de los desplazamientos angulares de las articulaciones del tobillo, rodilla y cadera, donde sólo se han obtenido claras diferencias entre las medias para la articulación de la cadera al final de la fase de absorción del impacto ( $\Delta \boldsymbol{\theta}$ CADERA (200); $\mathrm{p}<0.001)$. 
Gutiérrez-Dávila, M.; Pancorbo, D.; Olivares, J., y Rojas, F. J. (2018). Contribución de los brazos en el aterrizaje del salto vertical. RICYDE. Revista internacional de ciencias del deporte, 52(14), 163-173. https://doi.org/10.5232/ricyde2018.05206

Tabla 2.- Estadística descriptiva e inferencial de las componentes de los desplazamientos lineales del CG en el plano sagital y los desplazamientos angulares de las articulaciones del tobillo, rodilla y cadera hasta el instante en que se alcanza el 2-PMF) y la fase de absorción del impacto (200 ms tras el contacto con la plataforma), así como las componentes de la velocidad alcanzada por el CG en esos dos instantes.

\begin{tabular}{|c|c|c|c|c|c|}
\hline Instante & variables & sin brazos & con brazos & $\mathbf{f}$ & Cohen's d \\
\hline \multirow{7}{*}{ 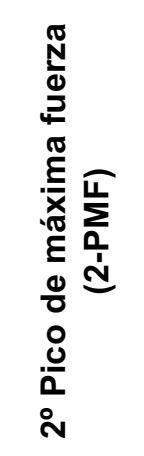 } & Posición vertical CG (m) & $0.136 \pm 0.027$ & $0.148 \pm 0.039$ & $4.24^{*}$ & 0.4 \\
\hline & Posición horizontal CG (m) & $0.017 \pm 0.003$ & $0.018 \pm 0.005$ & 3.00 & 0.1 \\
\hline & $\Delta \theta_{\text {TOBILLO }}\left({ }^{\circ}\right)$ & $39 \pm 11$ & $41 \pm 10$ & 0.67 & 0.2 \\
\hline & $\Delta \theta_{\text {RODILAA }}\left({ }^{\circ}\right)$ & $27 \pm 8$ & $29 \pm 9$ & 1.68 & 0.2 \\
\hline & $\Delta \theta_{\text {CADERA }}\left({ }^{\circ}\right)$ & $12 \pm 5$ & $14 \pm 6$ & 1.74 & 0.3 \\
\hline & v vertical CG $\left(\mathrm{m} \cdot \mathrm{s}^{-1}\right)$ & $-2.489 \pm 0.255$ & $-2.543 \pm 0.262$ & 1.47 & 0.2 \\
\hline & v horizontal CG $\left(\mathrm{m} \cdot \mathrm{s}^{-1}\right)$ & $0.351 \pm 0.062$ & $0.336 \pm 0.089$ & 0.72 & 0.2 \\
\hline \multirow{7}{*}{ 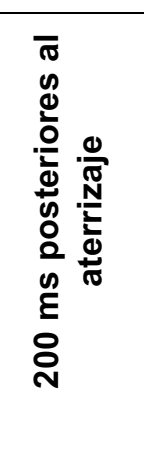 } & Posición vertical CG (m) & $0.349 \pm 0.060$ & $0.380 \pm 0.055$ & $7.89^{\star \star}$ & 0.5 \\
\hline & Posición horizontal CG (m) & $0.043 \pm 0.014$ & $0.037 \pm 0.015$ & $4.85^{\star}$ & 0.4 \\
\hline & $\Delta \theta_{\text {TOBILLO }}\left({ }^{\circ}\right)$ & $55 \pm 9$ & $52 \pm 9$ & 2.37 & 0.3 \\
\hline & $\Delta \theta_{\text {RODILAA }}\left({ }^{\circ}\right)$ & $80 \pm 11$ & $80 \pm 8$ & 0.03 & 0 \\
\hline & $\Delta \theta_{\text {CADERA }}\left({ }^{\circ}\right)$ & $58 \pm 10$ & $67 \pm 9$ & $28.18^{* * *}$ & 1.0 \\
\hline & v vertical CG $\left(\mathrm{m} \cdot \mathrm{s}^{-1}\right)$ & $-0.737 \pm 0.392$ & $-0.947 \pm 0.356$ & $10.25^{* *}$ & 0.6 \\
\hline & v horizontal CG $\left(\mathrm{m} \cdot \mathrm{s}^{-1}\right)$ & $-0.019 \pm 0.114$ & $-0.055 \pm 0.104$ & 2.56 & 0.4 \\
\hline
\end{tabular}

CG: centro de gravedad. $\Delta \theta$ : Incremento de ángulo o rango de movimiento. v: velocidad.

$$
{ }^{* * *} p<0.001 ;{ }^{* *} p<0.01 ;{ }^{*} p<0.05
$$

En la tabla 2 también se presentan las componentes de las velocidades del CG para el instante en que se produce el pico máximo de fuerza $\left(\boldsymbol{t}_{2-\mathrm{PMF}}\right)$ y al final de la fase de absorción del impacto. Con respecto a estas variables, no han existido diferencias entre la medias de las dos condiciones para el instante en que se produce el pico máximo de fuerza. Sin embargo, los datos relativos a la componente vertical de la velocidad al final de esta fase $\left(\mathbf{v y}_{\mathrm{CG}(200)}\right)$ confirman nuestra hipótesis, al constatarse que dicha velocidad es significativamente mayor cuando el aterrizaje se realiza con la participación de los brazos $\mathrm{p}<0.01$ ).

En la tabla 3 se presentan los datos de tendencia central sobre la contribución segmentaria durante la recepción, expresada en porcentajes del desplazamiento vertical del CG hasta $\boldsymbol{t}_{2-}$ PMF y el final de fase de absorción del impacto $(200 \mathrm{~ms})$, para las dos condiciones experimentales. Como se esperaba según el protocolo propuesto, ha existido una menor contribución de los brazos cuando éstos se restringen. Sin embargo, aunque los participantes han mantenido las manos en las caderas durante la recepción, ha existido una cierta contribución durante los primeros instantes $(0.96 \pm 0.53$, para Cbrazos-(2-PMF) $)$, posiblemente debido al movimiento de depresión de los hombros. Al final de $\mathbf{t}_{2 \text {-PMF }}$ sólo han existido ciertas diferencias estadísticamente significativas entre las medias para la contribución de los pies $(\mathrm{p}<0.05)$, siendo menor cuando la recepción se realiza con la participación libre de los brazos. Sin embargo, esta diferencia estaría condicionada por el desplazamiento vertical del $\mathrm{CG}$, ya que sus valores se expresan con respecto a su porcentaje. Así, como se ha indicado, los datos indican que no han existido diferencias en la posición angular de la articulación del tobillo en la toma de contacto ni en su desplazamiento angular posterior, lo que sugiere una 
Gutiérrez-Dávila, M.; Pancorbo, D.; Olivares, J., y Rojas, F. J. (2018). Contribución de los brazos en el aterrizaje del salto vertical. RICYDE. Revista internacional de ciencias del deporte, 52(14), 163-173. https://doi.org/10.5232/ricyde2018.05206

contribución neta similar para las dos condiciones. Sin embargo, al expresar este dato en porcentajes del desplazamiento vertical del $\mathrm{CG}$, su valor tiende a reducirse cuando aumenta el desplazamiento vertical, lo que ocurre cuando los aterrizajes se realizan con la participación libre de los brazos.

Tabla 3.- Estadística descriptiva e inferencial de las contribuciones segmentarias para las dos condiciones experimentales. Los datos se expresan en porcentaje con respecto al desplazamiento del CG hasta el segundo pico máximo de fuerza y el final de la fase de absorción del impacto $(200 \mathrm{~ms}$ tras el contacto con la plataforma).

\begin{tabular}{ccccc} 
variables & sin brazos & con brazos & $f$ & $\begin{array}{c}\text { Cohen's } \\
\text { d }\end{array}$ \\
\hline Cbrazos-(2-PMF) $(\%)$ & $0.96 \pm 0.53$ & $6.03 \pm 2.93$ & $79.72^{* * *}$ & 2.5 \\
Ctronco+cabeza-(2-PMF) $(\%)$ & $3.71 \pm 4.61$ & $4.07 \pm 4.28$ & 0.90 & 0.1 \\
Cmuslos-(2-PMF) (\%) & $18.34 \pm 12.32$ & $13.99 \pm 9.03$ & 2.23 & 0.4 \\
Cpiernas-(2-PMF) (\%) & $27.80 \pm 11.49$ & $31.89 \pm 10.42$ & 2.52 & 0.4 \\
Cpies-(2-PMF) $(\%)$ & $54.49 \pm 9.77$ & $48.77 \pm 12.86$ & $5.39^{*}$ & 0.6 \\
Cbrazos-(200) (\%) & $0.31 \pm 0.41$ & $8.88 \pm 3.68$ & $131.20^{* * *}$ & 4.2 \\
Ctronco+cabeza-(200) $(\%)$ & $10.64 \pm 4.43$ & $13.89 \pm 4.86$ & $10.05^{* *}$ & 0.7 \\
Cmuslos-(200) $(\%)$ & $38.64 \pm 8.00$ & $35.31 \pm 5.41$ & $6.72^{*}$ & 0.6 \\
Cpiernas-(200) $(\%)$ & $33.41 \pm 7.58$ & $28.21 \pm 6.91$ & $14.31^{* * *}$ & 0.8 \\
Cpies- $(200)(\%)$ & $16.61 \pm 6.48$ & $13.41 \pm 4.46$ & $8.04^{* *}$ & 0.6 \\
\hline$* * * \mathrm{p}<0.001 ;{ }^{* *} \mathrm{p}<0.01 ;{ }^{*} \mathrm{p}<0.05$ & & & &
\end{tabular}

Cuando se analiza la contribución de los segmentos al final de la fase de absorción del impacto, se puede generalizar que las diferencias entre las medias de las dos condiciones experimentales tienden a incrementarse hasta hacerse significativas. Así, la contribución media del tronco+cabeza es mayor con la participación libre de los brazos $(p<0.01)$, mientras que la contribución media de los muslos y las piernas es menor $(\mathrm{p}<0.05 ; \mathrm{p}<0.001$, para muslos y piernas respectivamente). Los valores reducidos de la contribución de los pies con respecto a los alcanzados en el segundo pico máximo de fuerza, indican que su contribución finalizó con el aterrizaje de su parte posterior con, lo que se asocia a 2-PMF.

\section{Discusión}

Los datos confirman la hipótesis inicial, al constatarse que la participación libre de los brazos contribuye a reducir del segundo pico máximo de fuerza (2-PMF) durante la recepción de los saltos verticales, el cual había sido asociado por diferentes autores a la producción de lesiones (McNair y col., 2000; Decker, y col., 2003; Cámara y col., 2013; Rojano y col., 2013). Los datos sugieren que la mayor reducción del 2-PMF con la acción libre de los brazos estaría motivada por un mayor desplazamiento vertical del CG desde el inicio hasta el instante en que se consigue el 2-PMF, debido a una posición más alta del CG en la toma de contacto (ver tabla 1). Además de la contribución de los brazos a la mayor altura del CG, los datos indican una posición más extendida del saltador en el contacto debido a una mayor extensión de la articulación de la cadera (ver tabla 1). En este sentido, se puede afirmar que una posición extendida y con los brazos elevados al inicio de los aterrizajes, incrementaría la distancia de frenado del CG con el mismo rango de movimiento articular durante el periodo inicial de la amortiguación (2-PMF; ver tabla 2) y, en consecuencia, se reduciría la magnitud de la fuerza de reacción vertical durante este periodo. 
Gutiérrez-Dávila, M.; Pancorbo, D.; Olivares, J., y Rojas, F. J. (2018). Contribución de los brazos en el aterrizaje del salto vertical. RICYDE. Revista internacional de ciencias del deporte, 52(14), 163-173. https://doi.org/10.5232/ricyde2018.05206

En contra de una de nuestras hipótesis iniciales, el hecho de no haber existido diferencias significativas entre las medias de la velocidad vertical del CG en el instante en que se produce 2-PMF ( $\left.\boldsymbol{v y}_{\mathrm{CG}(2-\mathrm{PMF})}\right)$, indica una escasa participación amortiguadora de los brazos desde el inicio del contacto hasta el segundo pico máximo de fuerza, lo que estaría relacionado con la escasa coordinación de la muestra para realizar la amortiguación durante este periodo inicial. Siguiendo a Gutiérrez-Dávila, Garrido, Amaro, \& Rojas (2014), en el caso de haber existido un desplazamiento activo de los brazos hacia abajo, cuando el CG también se está desplazando en el mismo sentido, la velocidad vertical del CG sería mayor en el segundo pico de fuerza con respecto a la situación donde se restringe la participación de los brazos y por tanto se reduciría el impulso de frenado. Por otra parte, esta escasa participación activa de los brazos durante la amortiguación se confirma al comprobar su reducida contribución al desplazamiento vertical del CG (6.03 \pm 2.93 ; ver tabla 3$)$. En este sentido, podríamos afirmar que una mayor aceleración de los brazos hacia abajo durante los primeros instantes de los aterrizajes, reduciría en menor grado la velocidad vertical y, en consecuencia, también la fuerza vertical que lo produce. Se sugiere que un proceso de aprendizaje de la técnica de recepción, donde se los brazos comiencen una aceleración hacia abajo instantes antes de tomar contacto con el suelo y ésta sea mantenida hasta el segundo pico máximo de fuerza, cuando el talón tome contacto con el suelo, que contribuiría a reducir la posibilidad del riesgo de lesiones debido a la mayor amortiguación de 2-PMF.

Al final de la fase de absorción del impacto, el desplazamiento vertical del CG sigue siendo mayor con la participación libre de los brazos, incrementándose aún más la significación entre las medias $(\mathrm{p}<0.01)$. Además de la comentada contribución de los brazos al desplazamiento del CG, es necesario mencionar la aportación a este hecho del mayor desplazamiento angular de la articulación de la cadera. Efectivamente, con la participación libre de los brazos existe un mayor desplazamiento articular de cadera $(\mathrm{p}<0.001$; ver tabla 2$)$, lo que contribuye a que también se haya constatado una mayor contribución del segmento tronco + cabeza al desplazamiento vertical del CG $(\mathrm{p}<0.01$; ver tabla 3$)$. La mayor velocidad vertical del CG al final de fase de absorción del impacto con la acción libre de los brazos $(\mathrm{p}<0.01$; ver tabla 2) indica que éstos siguen desplazándose hacia abajo, mediante una extensión de hombros, en el final de esta fase, contribuyendo así a la amortiguación de las fuerzas verticales de reacción. Sin embargo, las diferencias registradas para las contribuciones segmentarias al desplazamiento vertical del CG sugiere la existencia de dos modelos diferentes de recepción, posiblemente debido a la función estabilizadora que tendrían los brazos, durante la fase de absorción del impacto, como señalan Niu y col. (2013), una acción de brazos que facilitaría la mayor contribución del tronco+cabeza en la amortiguación de las fuerzas, mientras que se reduciría la contribución de los muslos y las piernas. Un cambio de modelo que contribuiría a reducir la tensión articular $y$, concretamente, la probabilidad de lesiones en el ligamento cruzado anterior de la rodilla.

En este sentido, consideramos relevante comentar la mayor contribución de las piernas y los muslos al desplazamiento vertical del CG cuando se restringe la participación de los brazos (Cpiernas-(200); Cmuslos-(200); $\mathrm{p}<0.001 ; \mathrm{p}<0.05$, respectivamente; ver tabla 3). La mayor contribución de las piernas al desplazamiento vertical del CG supone un mayor desplazamiento de éstas hacia delante con respecto a un eje transversal que pasaría por la articulación del tobillo y una mayor contribución de los muslos supone una mayor rotación hacia atrás de éstos a través de un eje transversal que pasaría por las articulaciones de la rodilla. Este mecanismo supondría el incremento de la tensión del ligamento cruzado anterior, cuya función principal es evitar el desplazamiento anterior de la tibia con respecto al fémur (Alm, Ekstrom y Gillquist, 1974; McNair y Marshall, 1994; Ericksen y col., 2013). 
Gutiérrez-Dávila, M.; Pancorbo, D.; Olivares, J., y Rojas, F. J. (2018). Contribución de los brazos en el aterrizaje del salto vertical. RICYDE. Revista internacional de ciencias del deporte, 52(14), 163-173. https://doi.org/10.5232/ricyde2018.05206

\section{Conclusiones}

1.- En general su puede afirmar que, con la participación libre de los brazos, se incrementa la absorción del impacto debido a un mayor desplazamiento vertical del CG, así como una menor reducción de su velocidad vertical durante la fase de absorción del impacto. En concreto, se reduce el pico máximo de fuerza relacionado con la producción de lesiones (2$\mathrm{PMF}$ ), lo que estaría motivado por una mayor distancia de frenado del CG debido a la posición más alta del CG el inicio de la amortiguación.

2.- La menor reducción de la velocidad vertical del CG al final de la fase de absorción del impacto, la mayor contribución del tronco+cabeza y el menor desplazamiento de las piernas hacia delante, nos hacen pensar en una función estabilizadora de los brazos que, junto a la mayor distancia de frenado, podría reducir el riesgo de lesiones en la articulación de la rodilla.

3.- Sugerimos la necesidad de establecer estrategias de aprendizaje orientadas a la mejora de las técnicas individuales de recepción de los saltos con el propósito de reducir el riesgo de lesiones.

4.- En otro ámbito, consideramos que, ante la evidencia de la función amortiguadora de los brazos durante las recepciones de los saltos verticales, debería existir un consenso en los protocolos utilizados cuando se investigan o evalúan acciones de absorción de los impactos.

\section{Referencias}

Alm, A.; Ekstrom, H., \& Gillquist J. (1974). The anterior cruciate ligament. Acta Orthopaedica Scandinavica, (suppl) 445, 3-49.

Cámara, J.; Calleja-González, J.; Martínez, R., y Fernández-López, J.R. (2013). The effect of basketball footwear on the vertical ground reaction force during the landing phase of drop jumps. Revista de Psicología Deportiva, 22(1), 179-182.

Chappell, J.D.; Yu, B.; Kirkendall, D.T., \& Garret, W.E. (2002). A comparison of knee kinetics between male and female recreational athletes in stop-jump tasks. The American Journal of Medicine, 30(2), 261-267.

https://doi.org/10.1177/03635465020300021901

Cortes, N.; Onate, J.; Abrantes, J.; Gagen, L.; Dowling, E., \& Van Lunen, B. (2007). Effects of gender and foot-landing techniques on lower extremity kinematics during drop-jump landings, Journal of Applied Biomechains, 23, 289-299.

https://doi.org/10.1123/jab.23.4.289

Dapena, J., \& Chung, C.S. (1988). Vertical and radial motions of the body during the take-off phase of high jumping. Medicine and Science in Sports and Exercise, 20, 290302.

https://doi.org/10.1249/00005768-198806000-00014

Decker, M.J.; Torry, M.R.; Wyland, D.J.; Sterett, W.I., \& Steadman, J.R. (2003). Gender differences in lower extremity kinematics, kinetics and energy absorption during landing. Clinical Biomechanics, 18, 662-669.

https://doi.org/10.1016/S0268-0033(03)00090-1

de Leva, P. (1996). Adjustments to Zatsiorsky-Seluyanovs segment inertia parameters. Journal of Biomechanics, 29, 1223-1230.

https://doi.org/10.1016/0021-9290(95)00178-6 
Gutiérrez-Dávila, M.; Pancorbo, D.; Olivares, J., y Rojas, F. J. (2018). Contribución de los brazos en el aterrizaje del salto vertical. RICYDE. Revista internacional de ciencias del deporte, 52(14), 163-173. https://doi.org/10.5232/ricyde2018.05206

Ericksen, H.M.; Gribble, P.A.; Pfile, K.R., \& Pietrosimone, B.G. (2013). Different modes of feedback and peak veltical ground reaction force during jump landing. A systematic review. Journal of Athletic Training, 48(5), 685-692.

https://doi.org/10.4085/1062-6050-48.3.02

Gutiérrez-Dávila, M.; Garrido, J.M.; Amaro, F.; Ramos, M., y Rojas, F.J. (2012). Método para determinar la contribución segmentaria en los saltos. Su aplicación en el salto vertical con contramovimiento. Motricidad. European Journal of Human Movement, 29, $1-21$

Gutiérrez-Dávila, M.; Garrido, J.M.; Amaro, F.J., \& Rojas, F.J. (2014): An analysis of two styles of arm action in the vertical countermovement jump, Sports Biomechanics, 12, 2, 135-143.

https://doi.org/10.1080/14763141.2014.910832

Lees, A. (1981). Methods of impact absorption when landing from a jump. Engineering in Medicine. 10, 207-211.

https://doi.org/10.1243/EMED_JOUR_1981_010_055_02

Lobietti, R.; Coleman, S.; Pizzichillo, E., \& Merni, F. (2010). Landing techniques in volleyball. Journal of Sports Sciences, 28(13), 1469-1476.

https://doi.org/10.1080/02640414.2010.514278

McNair, P. J.; Prapavessis, H., \& Callender, K. (2000). Decreasing landing forces: effect of instruction. British Journal of Sports Medicine, 34(4), 293-295.

https://doi.org/10.1136/bjsm.34.4.293

McNair P.J., \& Marshall R.N. (1994). Landing characteristics in subjects with normal and anterior cruciate ligament deficient knee joints. Archives of Physical Medicine and Rehabilitation. 75(5), 584-589.

McNintt-Gray, J. (2000). Subject specific coordination of two and one joint muscles during landings suggests multiple control criteria. Motor Control, 4, 1-44.

Newman, J. S., \& Newberg, A. H. (2010). Basketball injuries. Radiologic Clinics of North America, 48(6), 1095-1111.

https://doi.org/10.1016/j.rcl.2010.07.007

Niu, W.; Zhang, M., Fan, Y. \& Zhao, Q. (2013). Dynamic postural stability for double-leg drop landing, Journal of Sports Sciences, 31(10), 1074-1081.

https://doi.org/10.1080/02640414.2012.762600

Niu, W.; Feng, T.; Jiang, C., \& Zhang, M. (2014). Peak Vertical Ground Reaction Force during Two-Leg Landing: A Systematic Review and Mathematical Modeling. BioMed Research International, 2014, 1-10.

https://doi.org/10.1155/2014/126860

Olivares, J.; Pancorbo, D.; Gutiérrez-Dávila, M., y Rojas, F.J. (2016). Efecto de la acción posterior a la recepción sobre la amortiguación en la recepción de los saltos verticales. En García-López, J. y Ogueta-Alday, A. (Eds). Actas del XXXIX Congreso de la Sociedad Ibérica de Biomecánica y Biomateriales (pp. 53-54). León. Editoria Universidad de León.

Rojano, D.; Rodríguez, E., \& Berral, F.J. (2010). Analysis of the vertical ground reaction forces and temporal factors in the landing phase of a countermovement jump. Journal of Sports Sciences and Medicine, 9, 282-287.

Rowley, K.M., \& Richards, J.G. (2015). Increasing plantarflexion angle during landing reduces vertical ground reaction forces, loading rates and the hip's contribution to support moment within participants. Journal of Sports Sciences, 33(18), 1922-1931. https://doi.org/10.1080/02640414.2015.1018928 
Gutiérrez-Dávila, M.; Pancorbo, D.; Olivares, J., y Rojas, F. J. (2018). Contribución de los brazos en el aterrizaje del salto vertical. RICYDE. Revista internacional de ciencias del deporte, 52(14), 163-173. https://doi.org/10.5232/ricyde2018.05206

Sampello, M. (2005). Review of motor control mechanisms underlying impact absorption from falls. Gait and Posture, 21, 85-97.

https://doi.org/10.1016/j.gaitpost.2004.01.005

Sampello, M.; McDonagh, M.J.N., \& Challis, J.H. (2001). Visual and no visual control of landing movements in humans. Journal of Physiology, 537, 313-340.

https://doi.org/10.1111/j.1469-7793.2001.0313k.x

Saunders, N.W.; Hanson, N.; Koutakis, P.; Chaudhari, A.M., \& Devor, S.T. (2014). Landing ground reaction forces in figure skaters and non-skaters. Journal of Sports Sciences, 32(11), 1042-1049.

https://doi.org/10.1080/02640414.2013.877593

Wilk, K. E.; Briem, K.; Reinold, M.M.; Devine, K.M.; Dugas, J., \& Andrews, J. R. (2006). Rehabilitation of articular lesions in the athlete's knee. Journal of Orthopaedic and Sports Physical Therapy, 36(10), 815-827.

https://doi.org/10.2519/jospt.2006.2303

Winter, D. (1990). Biomechanics and motor control of human movement. New York, NY. John Wiley.

Zatsiorsky, V. M., \& Seluyanov, N. V. (1983). The mass and inertial characteristics of the main segments of the human body. In H. Matsui \& K. Kobayashi (Eds.), Biomechanics VIII-B (pp. 1152-1159). Champaign, IL. Human Kinetics. 